\title{
Labor Composition and Long-Run Employment in a Minimum-Wage Economy
}

William D. Robinson

\begin{abstract}
This article examines the long-run consequences of minimum wage regulation within the framework of a two-labor-sector growth model, where a minimum real wage is effective only in the "unskilled" labor sector. The assumption of heterogeneous labor makes possible a stable steady-state growth equilibrium. Conditions for the stability and existence of steady-state equilibrium are presented. A significant implication of the model is that selective immigration policies that encourage the influx of skilled workers would have the long-run effect of increasing the employment rate of unskilled labor, even in cases where the two labor sectors are nearly perfect substitutes.
\end{abstract}

Macroeconomic literature commonly focuses on a cyclical component of unemployment whose causes may include wage and price stickiness or temporary misconceptions on the part of workers about market conditions. A more permanent component of unemployment, resulting from minimum wage regulation, calls for a longer-run perspective. A long-run approach would go beyond viewing a minimum wage as a movement along a given demand-for-labor curve and would also consider shifts of labor demand over time due to capital formation.

This paper presents a simple model of capital formation and employment in an economy with a minimum real wage and two labor sectors. The primary focus is on circumstances in which the minimum wage is effective in the "unskilled" sector but not in the "skilled" sector. The use of more than one labor sector in the model is crucial if there is to be a steady-state employment rate other than the extremes of $100 \%$ or $0 \%$. The two-labor-sector approach provides a step towards realism for this reason, and also because one would not expect skilled and unskilled labor to be perfectly substitutable in practice.

William D. Robinson is an Economist in the Office of Statistical Standands, Energy Information Administration, U.S. Deparment of Energy, Washington, DC 20585. 
Solow's pioneering work in economic growth (1956) briefly considers a case of "rigid wages" in real terms, where it is concluded that the employment rate approaches zero if the saving rate is below a certain critical value, and approaches $100 \%$ if the saving rate is above that critical value; an assumption of homogeneous labor is used in the Solow model. Enhancements to growth theory in subsequent years have not been aimed at examining the significance of labor heterogeneity within the framework of a model with a minimum real wage. This paper shows that the introduction of multiple labor sectors can provide a stabilizing element to an economic growth model with a minimum wage, thereby offering a tool for studying long-run determinants of the employment rate of workers subject to a minimum wage.

Because the model has a long-run focus, the minimum wage is expressed in real terms to reflect an assumption that the government adjusts the nominal minimum wage over time as the price level changes. Aside from legally-imposed wage floors, another reason for assuming a minimum real wage in an economic model is that society might provide welfare assistance to persons with incomes below a certain "poverty level" in a manner that eliminates work incentives below a certain real wage.

With the introduction of a heterogeneous labor force, a major determinant of the employment rate of unskilled workers is the relative supplies of skilled and unskilled workers. In view of recent U.S. legislation designed to encourage the immigration of skilled professionals (Tuma and Watson 1990; Parker 1990), it is instructive to consider the effects of such policies on the employment rate of unskilled workers. In the short run, the result depends on the complementarity-substitutability relationship between the two labor sectors. However, the model demonstrates that in the steady state, a higher ratio of skilled to unskilled workers always results in an increase in the employment rate of unskilled workers, even in circumstances where skilled and unskilled labor are nearly perfect substitutes; this result occurs because the influx of skilled workers stimulates capital formation, and in the long run the combined effect of a higher capital stock along with more skilled labor increases the marginal productivity of unskilled labor for any given level of employment.

\section{The Model}

This section describes the model assumptions and structure, and develops a framework for analyzing the effects of various government policies and other influences on the steady-state employment rate of unskilled workers.

\section{Basic Assumptions, Model Structure, and Steady-State Stability}

Output is assumed to be a linearly homogeneous function of three inputs capital, skilled labor, and unskilled labor:

$$
Y=F(K, S, U)
$$


where $Y$ is real output, assumed to be a single consumption/investment good, and where the variables $K, S$, and $U$, respectively represent the amounts employed of capital, skilled labor, and unskilled labor. There are positive and diminishing marginal returns with respect to each input,

$$
\begin{gathered}
F_{i}>0, \\
F_{i i}<0, \quad i=K, S, U
\end{gathered}
$$

where subscripts denote partial derivatives. $F$ is concave (non-strictly) and has second partial derivatives which are continuous. Perfect market competition prevails.

Define the variables

$$
\begin{aligned}
& \hat{y} \equiv \frac{Y}{K} \\
& \hat{s} \equiv \frac{S}{K} \\
& \hat{u} \equiv \frac{U}{K} .
\end{aligned}
$$

It follows that

$$
\hat{y}=F(1, \hat{s}, \hat{u}) \equiv f(\hat{s}, \hat{a}) .
$$

Saving is a constant fraction $\delta$ of income. For simplicity it is assumed that there is no depreciation of capital. Consequently,

$$
\dot{K}=\delta F(K, S, U)
$$

where a dot above a variable denotes a derivative with respect to time. It follows that

$$
\frac{\dot{K}}{K}=\delta f(\hat{s}, \hat{u})
$$

Both types of labor are inelastically supplied at a given point in time. The model assumes that capital and skilled labor are fully employed and that the minimum wage applies only to the unskilled sector. Hence

$$
f_{\hat{u}}(\hat{s}, \hat{u})=w
$$

where $w$ is the minimum real wage. The unskilled sector, therefore, is not fully employed.

The assumptions imply that there is a function which expresses $\hat{u}$ in terms of $\hat{s}$ and $w$ such that $(6)$ is satisfied ${ }^{1}$ : 


$$
\hat{u}=\varphi(s, w) \text {. }
$$

Substituting (7) into (5) yields

$$
\frac{\dot{K}}{K}=G(\hat{s}, w) \equiv \delta f(\hat{s}, \varphi(\hat{s}, w)) .
$$

The $G$ function defined here is applicable only as long as the minimum wage is effective only in the unskilled sector, with the marginal productivity of unskilled workers equal to $w$.

Assume that the skilled labor supply grows at a constant proportionate rate $n$ :

$$
\frac{\dot{s}}{S}=n
$$

It follows that

$$
\frac{\dot{s}}{\hat{s}}=n-G(\hat{s}, w) .
$$

The stability of the system depends crucially upon whether $G$ is an increasing or decreasing function of $\hat{s}$ (for a given $w$ ). If $G$ is an upward sloping function, as shown in Figure 1, upper-right quadrant, then the long-run equilibrium value $\hat{s}^{*}$ is dynamically stable. For example, for values of $\hat{s}$ to the left of $\hat{s}^{*}, n$ exceeds $G$. Hence from (10), $\hat{s}$ is growing over time; this growth continues until $\hat{s}^{*}$ is reached. By similar reasoning, $\hat{s}$ also tends toward $\hat{s}^{*}$ if $\hat{s}$ is initially greater than $\hat{s}^{*}$. Hence $\hat{s}^{*}$ marks a steady-state equilibrium.

If the $G$ function were downward-sloping, then any steady state would clearly be dynamically unstable.

The stability condition can be expressed in a form that has a simple economic interpretation. It can be shown that ${ }^{2}$

$$
G_{s}=-\delta \frac{F_{U}^{2}}{F_{U U}}\left[\frac{\partial}{\partial U}\left(\frac{F_{s}}{F_{U}}\right)\right] .
$$

Since $F_{U U}$ is negative and $\delta$ is positive, it follows that the sufficient condition for stability of a steady state is satisfied if and only if the marginal rate of technical substitution of skilled labor for unskilled labor $\left(F_{S} / F_{U}\right)$ is an increasing function of $U$ (for given values of $K$ and $S$ ). ${ }^{3}$ A geometric interpretation of the stability condition is provided in Figures $2 \mathrm{a}$ and $2 \mathrm{~b}$. The curves represent isoquants for production functions given a fixed capital stock. Figure 2a shows a case where the marginal rate of technical substitution of $S$ for $U$ (the negative of the slope of an isoquant) increases as $U$ increases; this is a stable case. Figure $2 b$ shows an unstable case. 


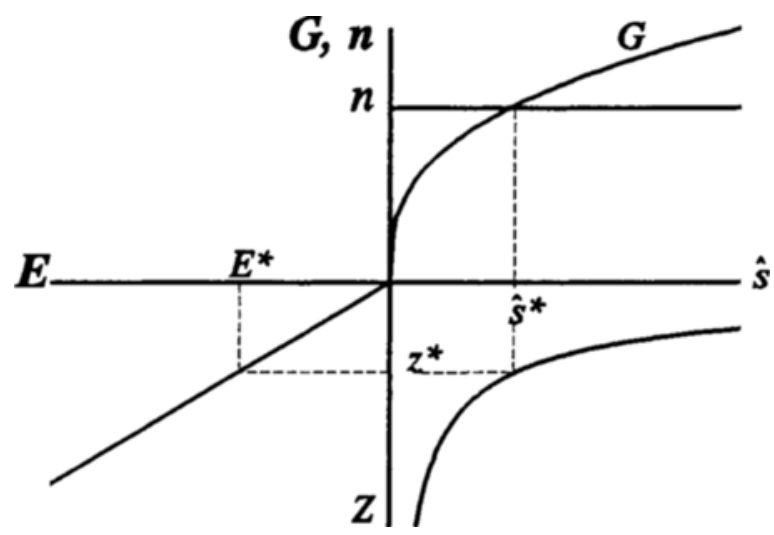

Fig. 1 .

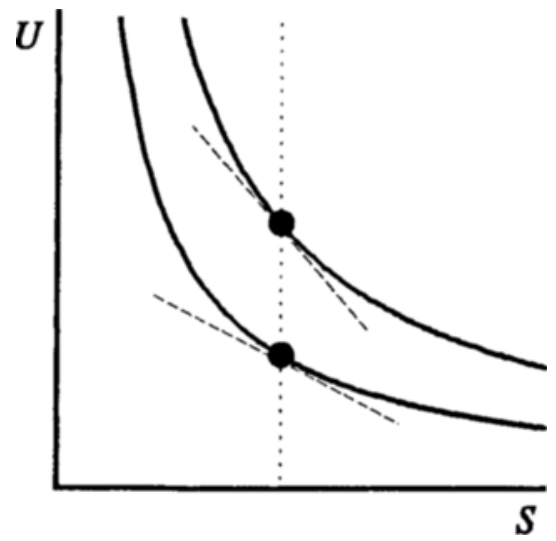

Fig. 2a. Stable Case: $F_{S} / F_{U}$ increases as $U$ increases.

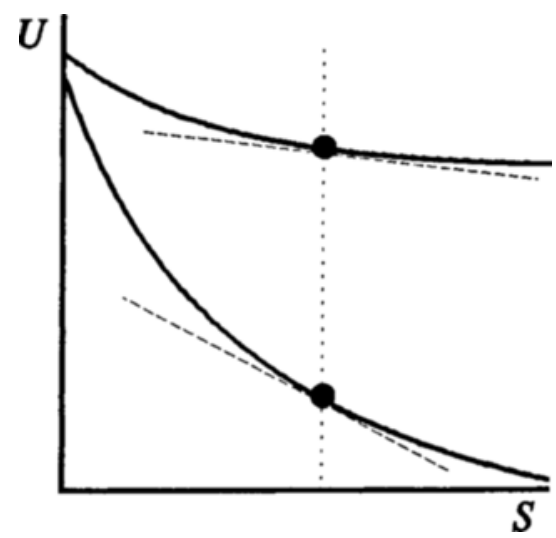

Fig 2b. Unstable Case: $F_{S} / F_{U}$ decreases as $U$ increases.

Of course, the stability condition just stated does not guarantee the existence of a steady state, for it is possible that the $G$ function might not intersect the line representing the growth rate of skilled labor. For this reason it will be useful to consider a specific production function later in the paper.

\section{Employment Rate Among Unskilled Workers}

In order to determine the employment rate of unskilled workers, it will prove useful to define the function 


$$
z(\hat{s}, w) \equiv \frac{\varphi(\hat{s}, w)}{s}=\frac{\hat{u}}{s}=\frac{U}{S} .
$$

Thus the $z$-function shows the ratio of unskilled labor employed to skilled labor as a function of $\hat{s}$ (given $w$ ). The slope of this function can be expressed as ${ }^{4}$

$$
z_{s}=\frac{1}{\hat{s}^{2}}\left[\frac{F_{U K}}{F_{U U}}\right] .
$$

Using the terminology of Ferguson $(1969,71) U$ and $K$ are said to be complementary inputs if $F_{U K}>0$. They are said to be competitive inputs if $F_{U K}<0$. Since $F_{U U}$ is negative, it follows that $z_{\hat{s}}$ is positive if capital and unskilled labor are competitive inputs; $z_{\hat{s}}$ is negative if capital and unskilled labor are complementary. The lower right quadrant of Figure 1 shows $z$ as a function of $\hat{s}$. In Figure 1 unskilled labor and capital are complementary inputs.

In order to make any kind of statement about the employment rate of unskilled workers, it is necessary also to specify an assumption about the available supply of unskilled workers. Assume that the skilled and unskilled labor supplies grow at the same proportionate rate:

$$
\frac{\dot{\tilde{U}}}{\tilde{U}}=\frac{\dot{S}}{S}=n .
$$

where $\tilde{U}$ denotes the available supply of unskilled workers. This assumption implies that the ratio of $S$ to $\tilde{U}$ remains constant. Let this ratio be denoted by $\Theta$ :

$$
\theta \equiv \frac{S}{\tilde{U}}
$$

Let $E$ denote the employment rate of unskilled workers:

$$
E \equiv \frac{U}{\tilde{U}}
$$

It follows that

$$
E=\theta \frac{U}{S}=\theta z
$$

This relationship is illustrated in the lower left quadrant of Figure 1. The slope of the line in that quadrant is determined by the value of $\theta$.

In Figure 1 , when the economy is at the steady-state value $\hat{s}^{*}$, the corresponding value of $z$ is $z^{*}$, and the employment rate among unskilled workers is $E^{*}$.

Recall that the $G$ function is applicable only in the range where $E \leq 1$. In a case where an increasing $G$ function intersects the $n$ line at a corresponding value of $E$ $>1$, the model dynamics would imply that whenever the economy is in the partial employment region $(E<1), E$ would increase until eventually full employment is 
reached. (This paper does not trace the dynamics of the model in the fullemployment region, since full-employment dynamics resembles traditional growth models (Solow 1956, 1988; Burmeister and Dobell 1970)).

It can be shown that in this model the marginal productivity of skilled labor is a nonincreasing function of $\hat{s}$ since $f(\hat{s}, \hat{u})$ is concave. ${ }^{5}$ Hence it is possible that for sufficiently large $\hat{s}$, skilled labor's wage could fall below the minimum wage. Nevertheless, for simplicity, the model assumes that the minimum wage is effective only in the unskilled sector. It would be understood that for a realistic application of the model, skilled labor's wage would remain above the minimum wage.

\section{An Example}

As an illustrative example, consider the class of CES production functions of the form

$$
F(K, S, U)=A K^{(1-\lambda)}\left[\gamma S^{-\beta}+(1-\gamma) U^{-\beta}\right]^{-\frac{\lambda}{\beta}}
$$

where $0<\lambda<1$ (Uzawa 1962). Our attention here is restricted to the case where $-1<\beta<0$ and $1 / 2<\gamma<1$. (The restriction that $1 / 2<\gamma$ is needed to ensure that skilled labor's marginal productivity is higher than that of unskilled labor near the perfect-substitutability case $\beta \Downarrow-1$.) It can be shown that the $G$ function intersects the vertical axis at a critical value which we will call $\Omega$, and is increasing and unbounded from above, as illustrated in Figure 3. The $z$ function is decreasing with respect to $\hat{s}$ (as in Figure 3 ) and asymptotically approaches each axis. ${ }^{6}$ Hence for sufficiently low $\hat{s}$, full employment of unskilled labor is attained. Under no circumstances does unskilled labor become completely unemployed. The latter property holds because when perfect labor substitutability is excluded-that is $\beta \neq 1$-then the marginal productivity of unskilled labor would become arbitrarily large as the employment of unskilled labor approaches zero, thereby precluding complete unemployment of unskilled workers. Furthermore, $E$ cannot even approach 0 asymptotically in this case: Since $G$ is unbounded from above, the $n$ line cannot lie entirely above the $G$ curve; this eliminates any possibility that $\hat{s}$ could grow arbitrarily large as $E$ approaches 0 .

Somewhere above $\Omega$ there is a critical labor growth rate $n_{f}$ which corresponds to an employment rate of 1 as shown in Figure 3. For $n>n_{f}$ the economy converges to a partial-employment steady state, with $0<E<1$. The $G$ and $z$ curves are applicable in this range. For $n<n_{f}$ the economy reaches full employment. 


\section{Change in the Relative Quantities of Skilled and Unskilled Workers}

This section considers the effect of a supposed one-time influx of skilled immigrants, with an assumption that the new higher ratio $\theta$ of skilled to unskilled workers is maintained in subsequent time periods as both labor sectors grow at the same proportionate rate. A possible rationale for the assumed permanency of the higher $\theta$ is that genetic and socioeconomic factors tend to make the skill level of offspring resemble that of the parents. Alternatively, the

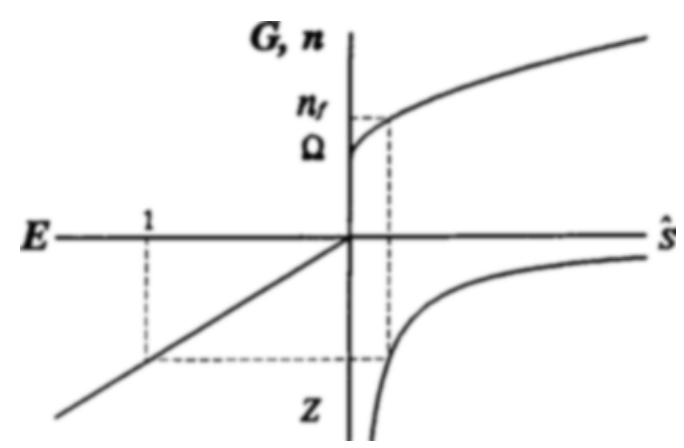

Fig. 3. CES Example for $-1<\beta<0$. For $n>n_{f}$ a steady state results with $0<E<1$. For $n<n_{f}$ full employment results. permanency of the higher $\theta$ could be a result of an ongoing government immigration policy that maintains this higher ratio at a fairly constant level.

In Figure 4, suppose that the economy is initially at a steady state equilibrium marked by $\hat{s}_{\mathrm{o}}$. The increase in the ratio of available skilled to unskilled labor has two effects in the short run: (1) the $E$-line in the lower left quadrant rotates clockwise, and (2) the short run $\hat{s}$ moves to a higher level, say $\hat{s}_{1}$. From the diagram it would appear that in the short run, the effect on unskilled employment could be either positive or negative. This is in fact the case, and it can be shown algebraically that the short-run effect on employment is positive if and only if skilled and unskilled labor are complementary. ${ }^{7}$

In the long run, however,

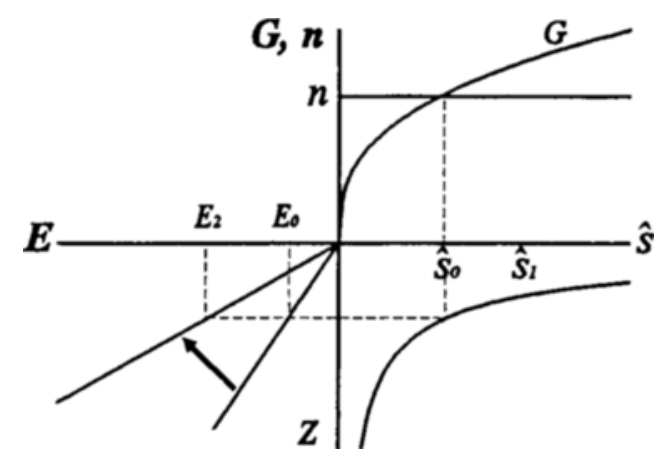

Fig. 4. Influx of skilled workers raises $\hat{s}$ only in short run. Employment rate of unskilled workers increases in long run. the $\hat{s}$ returns to the steady-state value $\hat{s}_{\mathrm{o}}$. The only permanent change in the diagram from the influx of skilled workers is the shift in the $E$-function in the lower-left quadrant. The steady-state employment rate has increased to $E_{2}$. Hence the influx of skilled workers unambiguously increases the employment rate of unskilled workers in the steady state, regardless of the complementary-substitutability relationship between the two 
labor types. This result occurs because the influx of skilled labor stimulates capital formation, and hence in the long run, the combined effects of higher skilled labor and capital augments the marginal productivity of unskilled labor.

These long-run results are applicable, of course, when there exists a stable steadystate equilibrium. It is significant that for the CES class discussed in the previous section, the conclusions are applicable even if skilled and unskilled labor are highly substitutable, excluding only the extreme case in which the two labor sectors are perfect substitutes (and excluding cases where all labor becomes fully employed).

There are, of course, other possible causes of a change in the ratio of skilled to unskilled workers. Educational and birth-control policies are examples. The steadystate results described above would be applicable when the policies are pursued in a way that a change in $\theta$ is permanently sustained.

\section{Other Applications of the Model}

Another application of the model is to consider the relative strengths of the shortrun and long-run effects on employment of an increase in the minimum real wage. ${ }^{8}$ "Short-run" refers here to a period of time in which the stocks of capital and labor are fixed, whereas "long-run" refers to the steady state. An increase in the minimum real wage causes the $G$ and $z$ curves to shift toward the horizontal axis (not shown). The short run effect is reflected in the shift of the $z$ function with $\hat{s}$ fixed at its initial level. The long run impact would also include the movement of $\hat{s}$ to a new steady state due to the shift of the $G$ curve. It can be seen that if capital and unskilled labor are complementary, represented by a decreasing $z$ function (as in Figures 1 and 3), then an increase in the minimum real wage would lead to a greater decrease in the employment rate in the long run than in the short run. But if capital and unskilled labor are competitive, then the effect is weaker in the long run than in the short run.

The model also demonstrates that the following results hold when capital and unskilled labor are complementary:

(1) An increase in the propensity to save leads to an increase in the steady-state unskilled employment rate.

(2) An increase in the rate of population growth of both labor sectors leads to a decrease in the steady-state unskilled employment rate.

The opposite conclusions hold if capital and unskilled labor are competitive factors.

\section{Conclusions and Suggestions for Future Research}

This paper has examined the implications of relaxing the assumption of perfect labor-force homogeneity in a growth model with a minimum real wage. The use of two labor sectors which are not perfectly substitutable provides a stabilizing element to the model which makes it possible to apply steady-state comparative statics analysis for determining the long-run impacts on employment of various government policies and other influences. 
A significant result is that an increase in the skilled labor supply relative to that of unskilled labor increases the steady-state employment rate of unskilled workers. That this conclusion is applicable to all cases except perfect substitutability-at least within the CES class considered-points to the loss of information that can occur when labor is treated as homogeneous in an economic model. The result implies that educational policies aimed at the skilled class provide long-run benefits to unskilled labor. The model also provides a case for policies designed to encourage the immigration of skilled labor.

The model has other applications, including the analysis of the relative strengths of short-run and long-run effects of an increase in the minimum wage.

A useful extension of the model would be the inclusion of a government spending sector. In particular, deficit spending and government borrowing can have significant impacts on the capital intensity of an economy, which would, in turn, affect steadystate employment. Finally, this model has not explicitly considered technological change, depreciation of capital, or more complex saving hypotheses. Such extensions remain useful topics for future research.

\section{Acknowledgement}

I am grateful to Mark Loewenstein, Meng Hua Ye, Robert Trost, Steve Suranovic, and two anonymous referees for helpful comments on earlier versions of this paper. Any remaining deficiencies are my sole responsibility.

\section{NOTES}

${ }^{1}$ Since $f_{\hat{u} \hat{u}}$ is nonzero, the existence of such an implicit function is guaranteed by the implicit function theorem since it is assumed that $f$ has continuous second partial derivatives.

${ }^{2}$ Equation (11) is derived by differentiating $G$ (Equation 8 ) with respect to $\hat{s}$, and by using implicit differentiation of (6) to obtain

$$
\varphi_{\hat{s}}=-f_{\hat{a} \hat{s}} / f_{\hat{a} \hat{a}},
$$

along with the facts that

$$
f_{\hat{s}}=F_{s}, \quad f_{\hat{u}}=F_{U}, \quad f_{\hat{a} \hat{s}}=K \cdot F_{U S},
$$

and

$$
f_{a \hat{u}}=K \cdot F_{U U}
$$

The derivation also uses the fact that

$$
F_{U S}=F_{S U}
$$

which holds because $F$ is assumed to be twice continuously differentiable. Using these conditions, it follows that 
from which (11) follows.

$$
G_{3}=-\delta \frac{F_{U}^{2}}{F_{U U}}\left[\frac{F_{U} F_{U S}-F_{S} F_{U U}}{F_{U}^{2}}\right]
$$

${ }^{3}$ There is some variation in the terminology used in economics literature: In this paper $F_{S} / F_{U}$ is referred to as the marginal rate of technical substitution of $S$ for $U$. Some books would use " $U$ for $S$ " to refer to the same mathematical expression.

${ }^{4}$ The derivation of (13) makes use of conditions described in note 2 , along with the fact that

$$
K \cdot F_{U K}+S \cdot F_{U S}+U \cdot F_{U U}=0
$$

since the production function is homogeneous degree one.

${ }^{5}$ This statement follows from the fact that

$$
\frac{\partial}{\partial \hat{s}} f_{s}(\hat{s}, \varphi(\hat{s}, w))=f_{s s}+f_{s \hat{Q}} \varphi_{s}=f_{s \hat{s}}-f_{s \hat{A}} \frac{f_{Q s}}{f_{\hat{A} \hat{Q}}}=\frac{1}{f_{\hat{A} \hat{Q}}}\left(f_{s s} f_{\hat{A} \hat{k}}-f_{R s}^{2}\right) \text {. }
$$

Since $f$ is concave

$$
f_{\hat{A} \hat{A}} \leq 0 \text { and } f_{\hat{B} s} f_{\hat{A} \hat{A}}-f_{\hat{A s}}^{2} \geq 0 \text {, }
$$

and hence $\frac{\partial}{\partial \hat{s}} f_{s}$ is negative or zero.

${ }^{6}$ Algebraic derivations for this example will be made available from the author upon request.

${ }^{7}$ This fact can be seen by differentiating the equation $F_{U}(K, S, U)=w$ along with equation (16). Then by setting $d w=d K=0$ and solving, one obtains

$$
d E=\frac{1}{\tilde{U}}\left(-\frac{F_{U S}}{F_{U U}}\right) d S .
$$

Since $F_{U U}$ is negative, the stated short-run result follows.

${ }^{8}$ Surveys of literature on the unemployment effects of minimum wage regulation are presented by Brown, Gilroy, and Kohen (1982), and Brown (1988).

\section{REFERENCES}

Brown, Charles. "Minimum Wage Laws: Are They Overrated?" Journal of Economic Perspectives 2 (Summer 1988): 133-45.

Brown, Charles, Curtis Gilroy, and Andrew Kohen. "The Effect of the Minimum Wage on Employment and Unemployment." Journal of Economic Literature 20 (June 1982): 487-528.

Burmeister, Edwin, and A. Rodney Dobell. Mathematical Theories of Economic Growth. New York: Macmillan, 1970. 
Ferguson, C.E. The Neo-Classical Theory of Production and Distribution. Cambridge: Cambridge University Press, 1969.

Parker, Maynard, ed. "Give Me Your Smart, Your Skilled..." Newsweek 116 (17 December 1990): 33.

Solow, Robert M. "A Contribution to the Theory of Economic Growth." Quarterly Journal of Economics 70 (February 1956): 65-94.

. Growth Theory: An Exposition. New York: Oxford University Press, 1988.

Tuma, Clara, and Tom Watson. "Immigration Act Boon to Firms With Elite Clients." Legal Times 13 (17 December 1990): 6-7.

Uzawa, Hirofumi. "Production Functions with Constant Elasticities of Substitution." Review of Economic Studies 29 (October 1962): 291-99. 